\title{
PRIME $z$-FILTERS ON COMPLETELY REGULAR SPACES $\left({ }^{1}\right)$
}

\author{
BY \\ CARL W. KOHLS
}

\begin{abstract}
A prime $z$-filter on a space $X$ is a proper prime dual ideal in the lattice $Z(X)$ of zero-sets on $X$; with no loss of generality, one may take the space to be completely regular $[3,3.9]$. Each prime $z$-filter is contained in a unique maximal $z$-filter ( $z$-ultrafilter), and thus is associated with a unique point of the StoneCech compactification $\beta X$. Prime $z$-filters are useful tools in both topological and algebraic investigations. For example, the fact that primeness is, in a certain sense, preserved by continuous mappings is used by Gillman and Jerison to prove the theorem that an arbitrary product of realcompact spaces is realcompact $[3,8.11]$. The fact that the natural correspondence between $z$-filters on $X$ and ideals in the ring of continuous functions $C(X)$ preserves primeness is used many
\end{abstract} times in the study of the prime ideal structure of $C(X)$.

In [5], a technique was developed for describing prime $z$-filters on a completely regular space that are contained properly in a $z$-ultrafilter associated with a $G_{\delta}$-point $p \in X$. Essentially, one considers the prime $z$-filters on $X-\{p\}$ (especially the $z$-ultrafilters) each of whose sets has $p$ as a limit point. The case in which the $z$-ultrafilters are used enables one, on specific spaces, to find easily prime $z$-filters whose existence does not seem to be readily evident otherwise.

The goal of the present investigation was to improve this technique so that the description would be in terms of $z$-ultrafilters on suitable spaces, for all prime $z$-filters contained properly in the given $z$-ultrafilter, and to generalize its applicability to all points. Because the set of prime $z$-filters containing a given one form a chain $[5$, p. 448], and every $z$-filter containing a prime $z$-filter is also prime $[3,2.9]$, it is relatively simple to describe prime $z$-filters containing a given one; so a method of description is of interest primarily for prime $z$-filters contained properly in a given one. We have succeeded in providing a construction for prime $z$-filters contained properly in a certain type of prime $z$-filter that has an immediate successor, and also in obtaining a sufficient condition for a prime $z$-filter with an immediate successor to be minimal. In completely normal spaces,

Received by the editors November 20, 1963.

(1) This research was supported by the National Science Foundation, under grant NSF G-20747. The referee's many valuable suggestions and corrections resulted in a substantial improvment in the original exposition; his help is gratefully acknowledged. 
this construction is applicable to all nonminimal prime $z$-filters with an immediate successor, and the condition for minimality is also necessary; whether these statements are true in more general spaces is left open.

We have no corresponding results in the case of a prime $z$-filter without an immediate successor. Examples are provided to show that this case can be very complicated-one of them answers affirmatively the question of Gillman and Jerison on the existence of a prime $z$-filter that has no immediate successor and is not the intersection of a countable family of larger $z$-filters $[2, p$. 425].

Most of the requisite background material may be found in the paper [2] and book [3] of Gillman and Jerison. In addition, the reader should be familiar with the main ideas of [5] and with the space defined in $[4,4.2]$.

The notation and terminology used in the paper generally coincide with that of [3].

1. Prime $z$-filters with an immediate successor. At first glance, it may seem that the construction given in [2, Theorem 3.5] completely solves the problem of finding a prime $z$-filter contained properly in a given one. However, the following simple example shows that this is not the case. Let $E$ be the space introduced in $[1,8.5] ; E$ consists of a denumerable discrete space $N$ together with one point $e \in \beta N-N$, called the $\beta$-point. Let $\mathscr{U}$ be the free ultrafilter corresponding to $e$. To each isolated point of one copy $E_{1}$ of $E$, we attach another copy $E_{2}$ of $E$ by identifying the $\beta$-point of $E_{2}$ with the isolated point. The resulting space is denoted by $E^{(2)}$. Let $\mathscr{P}$ be the prime $z$-filter of zero-sets on $E^{(2)}$ that contain a set in the ultrafilter $\mathscr{U}$ on $E_{1}-\{e\}$. Suppose one tried to construct a prime $z$-filter $\mathscr{Q}$ as in $[2,3.5]$ so that $\mathscr{P}$ is the immediate successor of $\mathscr{2}$; one would, of course, pick $A \in \mathscr{P}$ (for instance, $A=E_{1}$ ). Now unless $\mathscr{M}$ were chosen judiciously, the prime $z$-filter $\mathscr{Q}$ obtained would not be contained in $\mathscr{P} ; \mathscr{Q}$ might have sets disjoint from certain sets in $\mathscr{P}$ that are subsets of $A$.

It is desirable to have a method that always yields a suitable $\mathscr{Q}$. The technique employed in [2, Example 4.3], although on a special space, indicates the correct direction in which to proceed. We now give a general construction that serves our purposes. In order to include the case of a $z$-ultrafilter, it is convenient to use the convention that a $z$-ultrafilter on a space $X$ has an immediate successor, namely, $Z(X)$.

THEOREM 1. Let $\mathscr{P}$ and $\mathscr{R}$ be prime z-filters on the space $X$, with $\mathscr{R}$ the immediate successor of $\mathscr{P}$ (possibly $\mathscr{R}=Z(X)$ ); assume there exist $A \in \mathscr{R}-\mathscr{P}$ and $B \in \mathscr{P}$, with $A \subset B$, such that $\mathrm{cl}_{(X-A)}(X-B)$ meets every set of $\mathscr{P}$ on $X-A$. Let $\mathscr{S}$ denote the trace of $\mathscr{P}$ on $X-A ; \mathscr{T}$, the collection of all zero-set neighborhoods in $X-A$ of sets in $\mathscr{S}$; $\mathscr{U}$, any $z$-ultrafilter on $X-B$ containing the trace of $\mathscr{T}$ on $X-B$; and $\mathscr{Q},\{Z \in Z(X): Z-B \in \mathscr{U}\}$. Then $\mathscr{Q}$ is a prime $z$-filter on $X$ contained properly in $\mathscr{P}$. 
Proof. First we show that $\mathscr{T}$ is a base for a $z$-filter on $X-A$. Since $A \notin \mathscr{P}$, we have $\varnothing \notin \mathscr{S}$, whence $\varnothing \notin \mathscr{T}$. If $Z_{1}$ and $Z_{2}$ belong to $\mathscr{T}$, then there exist $W_{1}$ and $W_{2}$ in $\mathscr{P}$ such that $Z_{i}$ is a zero-set neighborhood of $W_{i}-A(i=1,2)$. Now $Z_{1} \cap Z_{2}$ is a zero-set, and the interior of $Z_{1} \cap Z_{2}$ is the intersection of the interiors of $Z_{1}$ and $Z_{2}$. Hence $Z_{1} \cap Z_{2}$ is a zero-set neighborhood of the set $\left(W_{1}-A\right) \cap\left(W_{2}-A\right)=\left(W_{1} \cap W_{2}\right)-A$ in $\mathscr{S}$; so $Z_{1} \cap Z_{2} \in \mathscr{T}$.

For each $Z \in \mathscr{P}$ we can find a point $x \in(Z-A) \cap \mathrm{cl}_{(X-A)}(X-B)$, so that every neighborhood of $x$ meets $X-B$, whence a fortiori every zero-set neighborhood of $Z-A$ meets $X-B$; thus every set in $\mathscr{T}$ meets $X-B$. It follows immediately that the trace of $\mathscr{T}$ on $X-B$ is also a base for a $z$-filter, so that there actually is at least one $z$-ultrafilter $\mathscr{U}$ containing it. Finally, it is easy to verify that $\mathscr{Q}$ is a prime $z$-filter on $X$ (cf. [3, 4.12]).

We wish to show that $\mathscr{Q} \subset \mathscr{P}$. Let $Z \in \mathscr{Q}$, and suppose that $Z \notin \mathscr{P}$. Then $\mathscr{P} \cup\{Z\}$ generates a $z$-filter that contains $\mathscr{P}$ properly, and thus contains $\mathscr{R}$, and hence in particular the set $A$. So there exists a $Z^{\prime} \in \mathscr{P}$ such that $Z^{\prime} \cap Z \subset A$. Since $Z-A$ and $Z^{\prime}-A$ are disjoint, they are completely separated in $X-A$; thus, there exists a zero-set neighborhood $W$ of $Z^{\prime}-A$ disjoint from $Z-A$, and hence from $Z-B$. Now $Z^{\prime} \in \mathscr{P}$, so $Z^{\prime}-A \in \mathscr{S}$ and $W \in \mathscr{T}$. Also $Z-B \in \mathscr{U l}$ because $Z \in \mathscr{Q}$. On the other hand, $W-B$, which is in $\mathscr{U}$, is disjoint from $Z-B$. This is impossible; therefore $\mathscr{Q} \subset \mathscr{P}$. Because $B \in \mathscr{P}-\mathscr{Q}$, the inclusion is proper.

REMARK 1. In case $\mathscr{P}$ is a $z$-ultrafilter, one may as well always choose $A$ to be empty; if the hypothesis in the theorem is satisfied at all, it will be satisfied in this case. Thus the condition reduces to: There exists $B \in \mathscr{P}$ such that $\operatorname{cl}_{X}(X-B)$ meets every set of $\mathscr{P}$.

REMARK 2. As a special case of the hypothesis that is simpler and more natural, one can require that $B-A$ be nowhere dense in $X-A$. However, this misses some familiar examples. For instance, the theorem is applicable in case $X$ is the one-point compactification of an uncountable discrete space and $\mathscr{P}$ is the $z$-ultrafilter converging to the nonisolated point; one can choose $B$ to be any set in $\mathscr{P}$ with infinite complement. But there is no way of picking $B$ so that $B-A$ is nowhere dense in $X-A$ (even for nonempty $A$ ), since $B-A$ must contain an isolated point.

A sufficient condition will now be given for a prime $z$-filter with an immediate successor to be minimal. We have succeeded in proving the necessity of the condition for completely normal spaces; it follows from the sufficiency and the proof of the necessity that the construction of Theorem 1 can be applied in a completely normal space to any nonminimal prime $z$-filter with an immediate successor. Although the status of the necessity and of the construction of Theorem 1 is not settled for other spaces, note that it is the more common spaces, such as metric spaces, whose prime $z$-filters have generally been the least understood. 
THEOREM 2. Let $\mathscr{P}$ and $\mathscr{R}$ be prime $z$-filters on $X$, with $\mathscr{R}$ the immediate successor of $\mathscr{P}$. Then $\mathscr{P}$ is a minimal prime $z$-filter if for every $A \in \mathscr{R}-\mathscr{P}$, the following condition is satisfied:

For each $B \in \mathscr{P}$ with $A \subset B$, there exist $Z \in \mathscr{P}$ and a cozero-set $C$ on $X$ such that $Z-A \subset C-A \subset B-A$.

When $X$ is completely normal, and $\mathscr{P}$ is minimal, then the condition is satisfied for every $A \in \mathscr{R}-\mathscr{P}$.

Proof. Assume first that $X$ is completely normal, and that condition (*) is not satisfied for some $A \in \mathscr{R}-\mathscr{P}$. For convenience, we set $\beta(X-A)=T$. By the remark following 3.1 in [2], every cozero-set on $X-A$ is a cozero-set on $X$. Thus, there exists $B \in \mathscr{P}$ such that for each $Z \in \mathscr{P}, \operatorname{cl}_{T}(B-A)$ is not a neighborhood of $\mathrm{cl}_{T}(Z-A)[3,7.14]$; so $\mathrm{cl}_{T}(Z-A)$ meets $\mathrm{cl}_{T}\left(T-\mathrm{cl}_{T}(B-A)\right)$. Since $X-A$ is normal, disjoint closed sets have disjoint closures in $T$. But $\operatorname{cl}_{T}\left(\operatorname{cl}_{(X-A)}(X-B)\right) \cup \mathrm{cl}_{T}(B-A)=T$, so $Z-A$ meets $\operatorname{cl}_{(X-A)}(X-B)$. It follows from Theorem 1 that $\mathscr{P}$ is not minimal.

Now assume that condition (*) is satisfied for every $A \in \mathscr{R}-\mathscr{P}$, and let $\mathscr{F}$ be a $z$-filter contained properly in $\mathscr{P}$. Choose a fixed $B \in \mathscr{P}-\mathscr{F}$, and $A \subset B$ with $A \in \mathscr{R}-\mathscr{P}$. Let $Z$ and $C$ be as in condition (*), and set $Z^{\prime}=X-C$. Then $Z^{\prime} \in Z(X)$, and $Z^{\prime} \notin \mathscr{P}$, because $Z^{\prime}-A$ is disjoint from a set in the trace of $\mathscr{P}$ on $X-A$, namely, $Z-A$. Thus $Z^{\prime} \notin \mathscr{F}$. On the other hand, we have $B \notin \mathscr{F}$ by assumption. But $Z^{\prime} \cup B \supset(X-B) \cup B=X$, so $Z^{\prime} \cup B \in \mathscr{F}$. Hence $\mathscr{F}$ is not prime. Therefore $\mathscr{P}$ is minimal.

Corollary. If for every $A \in \mathscr{R}, P-A$ is open in $X-A$, for each $P$ in a base for $\mathscr{P}$, then $\mathscr{P}$ is minimal.

Proof. For every $A \in \mathscr{R}$, there is a $P \in \mathscr{P}$ corresponding to each $B \in \mathscr{P}$ such that $P \subset B$ and $P-A$ is open in $X-A$ (and hence is a cozero-set in $X-A$ ). Thus, condition $\left(^{*}\right)$ is satisfied.

As an example of how the corollary may be applied, let $X$ be the Tychonoff plank, $\mathscr{R}$ the $z$-ultrafilter $Z\left[M^{\left(\omega_{1} \cdot \omega_{0}\right)}\right]$, and $\mathscr{P}$ any prime $z$-filter contained properly in $\mathscr{R}$. It follows immediately from the corollary that $\mathscr{P}$ is minimal (cf. $[3,14 \mathrm{H}])$.

2. Prime $z$-filters without an immediate successor. This section has three purposes: (1) To indicate by two examples a difficulty encountered in seeking theorems analogous to those in $\S 1$ for the case of prime $z$-filters without an immediate successor (Examples 1 and 3); (2) to clarify the example first given in $[4,4.2]$, which as pointed out in the review of that paper, and in [2], is erroneous (Examples 1 and 2); (3) to answer a question of Gillman and Jerison-they present an example $[2,4.3]$ of a decreasing $\omega$-sequence of prime $z$-filters, and ask, in 
effect, whether there exists a decreasing sequence with no countable coinitial subset. We give here an example of such a sequence (Example 4). Since the algebraic problem studied in [2] was not solved for a sequence of this type, its existence shows that the problem really has not been settled completely.

The following fact will be utilized several times in this section [3, 14F.1]: A $z$-filter $\mathscr{F}$ is prime if and only if there exists an ultrafilter $\mathscr{V}$ such that the family of zero-sets in $\mathscr{V}$ is precisely $\mathscr{F}$.

Another useful result is the following.

Proposition 1. Let $\mathscr{J}$ be an ultrafilter on a set $A$, and $\mathscr{K}$ an ultrafilter on a set $B$. Let $\mathscr{L}$ be the family of subsets of $A \times B$ having the form $\bigcup_{a \in J}\{a\} \times K_{a}$, where $J \in \mathscr{J}$ and $K_{a} \in \mathscr{K}$ for each a. Then $\mathscr{L}$ is a base for an ultrafilter on $A \times B$.

Proof. It is easy to see that $\mathscr{L}$ is a base for a filter. Given a set $D \subset A \times B$, and any $a \in A$, define $D_{a}$ to be $\{b \in B:(a, b) \in D\}$; and set $D^{\prime}=\left\{a \in A: D_{a} \in \mathscr{K}\right\}$. Then either $D^{\prime} \in \mathscr{J}$ or $A-D^{\prime} \in \mathscr{J}$. In the first case, $D$ contains a set in $\mathscr{L}$; in the second case, $(A \times B)-D$ contains a set in $\mathscr{L}$.

In the rest of the paper, the symbol $\mathscr{U}$ will be reserved for the free ultrafilter corresponding to the $\beta$-point $e$ in the space $E$ used in the example preceding Theorem 1. We recall the definition of the space $E^{(\omega)}$ of $[4,4.2]$ : Starting with one copy $E_{1}$ of $E$, we attach to each isolated point of $E_{1}$ another copy $E_{2}$ of $E$ by identifying the $\beta$-point of $E_{2}$ with the isolated point. We now attach to each of the isolated points in the adjoined spaces still another copy of the space $E$, and repeat this process a countably infinite number of times. The spaces added at the $(n-1)$ st stage are denoted generically by $E_{n}$. The $\beta$-point of $E_{1}$ is still denoted by $e$. A basic neighborhood of $e$ is the union of a neighborhood of $e$ in $E_{1}$ with neighborhoods of the $\beta$-points in the spaces $E_{2}$ which lie in this neighborhood, and so on, through all the spaces $E_{n}$, for all $n$. The basic neighborhoods of any other point are defined in a similar manner. It is easy to see that the basic neighborhoods described are open-and-closed (and hence zero-set) neighborhoods; from this it follows immediately that $E^{(\omega)}$ is a completely regular space.

Let $E_{n}^{*}$ be the generic symbol for the set of all points in $E_{n}$ other than the $\beta$-point. In Example 1, we shall require the following special definition and proposition about zero-sets containing $e$.

Given $Z \in Z\left[M_{e}\right]$, we let $H(Z)$ be the set containing $Z$ such that $H(Z) \cap E_{n}^{*}=E_{n}^{*}$ whenever $Z \cap E_{n}^{*} \in \mathscr{U}$, and $H(Z) \cap E_{n}^{*}=Z \cap E_{n}^{*}$ whenever $Z \cap E_{n}^{*} \notin \mathscr{U}$. It is easy to see that $H(Z)$ is a closed set; since $E^{(\omega)}$ is a countable normal space, $H(Z)$ is therefore a zero-set. We shall say that $Z$ is full in case $H(Z)=Z$. Clearly $H(H(Z))=H(Z)$. Thus, the full sets are precisely those of the form $H(Z)$.

Proposition 2. Each z-filter $\mathscr{F}$ containing $Z\left[O_{e}\right]$ is determined by its full sets, in the sense that $H(Z) \in \mathscr{F}$ if and only if $Z \in \mathscr{F}$. 
Proof. Trivially, $Z \in \mathscr{F}$ implies that $H(Z) \in \mathscr{F}$. Now assume that $H(Z) \in \mathscr{F}$, and construct a set $W$ as follows: Start with $Z$, and add a deleted basic neighborhood, disjoint from $Z$, of each point of $Z \cap E_{1}$ such that for the $E_{2}$ attached to it, $Z \cap E_{2}^{*} \notin \mathscr{U}$; treat each $E_{2}$ such that $Z \cap E_{2}^{*} \in \mathscr{U}$ in the way that $E_{1}$ just was, and so on, for all $n$. Then $W$ is the union of a basic neighborhood $N$ of $e$ and $\left(E^{(\omega)}-N\right) \cap Z$; since $N$ is open-and-closed, $W \in Z\left(E^{(\omega)}\right)$. Also, because $W \supset N$, we have $W \in Z\left[O_{e}\right]$, whence $W \in \mathscr{F}$. Furthermore, $H(Z) \cap W=Z$; because if $Z \cap E_{n}^{*} \in \mathscr{U}$, then $Z \cap E_{n}^{*}=W \cap E_{n}^{*} \subset E_{n}^{*}=H(Z) \cap E_{n}^{*}$, while if $Z \cap E_{n}^{*} \notin \mathscr{U}$, then $H(Z) \cap E_{n}^{*}=Z \cap E_{n}^{*} \subset W \cap E_{n}^{*}$. Hence $Z \in \mathscr{F}$.

It will be convenient to "coordinatize" the space $E^{(\omega)}$. The set $E^{(\omega)}$ can be put into one-to-one correspondence with the set of all $\omega$-tuples of non-negative integers that have a finite number of nonzero coordinates, which are consecutive and begin with the 0 th. The point $e$ corresponds to $(0,0, \cdots, 0, \cdots), E_{1}$ corresponds to $\{(k, 0, \cdots, 0, \cdots): k<\omega\}$, and each $E_{n}(n \geqq 2)$ corresponds to

$$
\left\{\left(p_{1}, \cdots, p_{n-1}, k, 0,0, \cdots\right): k<\omega\right\},
$$

where $p_{1}, \cdots, p_{n-1}$ is some collection of positive integers.

When the error in $[4,4.2]$ was pointed out, it was not observed that it is still possible to obtain a decreasing $\omega$-sequence of prime $z$-filters on $E^{(\omega)}$; such a sequence is exhibited in Example 1. (The example given in $[2,4.3]$ is more complicated.)

EXAMPle 1. A nonminimal prime z-filter that is the intersection of a decreasing $\omega$-sequence. The $\omega$-sequence of prime $z$-filters defined here will be referred to repeatedly in the rest of the paper. Let $\mathscr{B}_{0}$ consist of the zero-set $\{e\}$, and for each positive $n<\omega$, let $\mathscr{B}_{n}$ be the collection of all zero-sets that are the intersection of a basic neighborhood of $e$ in $E^{(\omega)}$ and the union of all the $E_{k}$ 's with $k \leqq n$; then $\mathscr{B}_{n}$ has the finite intersection property, and thus generates a $z$-filter $\mathscr{F}_{n}$. (Note that $\mathscr{F}_{0}=Z\left[M_{e}\right]$.) Since $\mathscr{F}_{n}$ contains the prime $z$-filter $Z\left[\boldsymbol{O}_{e}\right]$, it is prime $[3,2.9]$; however, for $n>0$, we wish to give an independent proof here that will serve as a model for other proofs. Applying Proposition 1 inductively, we find that for each $n>0$, the trace of the family of neighborhoods of $e$ on the union of all the $E_{n}^{* \text { 's }}$ is an ultrafilter $\mathscr{V}_{n}$ on that set. As is easily seen, $\mathscr{V}_{n}$ generates an ultrafilter $\mathscr{W}_{n}$ on $E^{(\omega)}$. Now $\mathscr{W}_{n} \cap Z\left(E^{(\omega)}\right)$ is the $z$-filter of zero-sets on $E^{(\omega)}$ that contain a set in $\mathscr{V}_{n}$; so $\mathscr{B}_{n}$ generates $\mathscr{W}_{n} \cap Z\left(E^{(\omega)}\right)$. Thus, we have $\mathscr{W}_{n} \cap Z\left(E^{(\omega)}\right)$ $=\mathscr{F}_{n}$. Therefore $\mathscr{F}_{n}$ is prime. (A direct proof that $Z\left[O_{e}\right]$ is prime can also be obtained by use of the ultrafilters $\mathscr{V}_{n}$. It suffices to show that if $Z$ and $Z^{\prime}$ are zero-sets such that $Z \cap Z^{\prime}=E^{(\omega)}$ and $Z \notin Z\left[O_{e}\right]$, then $Z^{\prime} \in Z\left[O_{e}\right][3,2 \mathrm{E}]$. Observe that $Z^{\prime}$ contains the closure of the open set $E^{(\omega)}-Z$. If for each $n>0$, the intersection of the union of all the $E_{n}^{*}$ 's with $Z$ is in $\mathscr{V}_{n}$, then it can be deduced from the assumption $Z \notin Z\left[O_{e}\right]$ that $Z^{\prime}$ contains a set in the ultrafilter $\mathscr{U}$ on $E_{1}^{*}$; from this and the other information available, it is easy to see that $Z^{\prime} \in Z\left[O_{e}\right]$. On the 
other hand, if for some $n$, the intersection of the union of all the $E_{n}^{*}$ 's with $Z$ is not in $\mathscr{V}_{n}$, the fact that $\mathscr{V}_{n}$ is an ultrafilter yields the desired conclusion.)

Since the $z$-filter generated by each $\mathscr{B}_{n}$ contains $\mathscr{B}_{n+1}$, the $\mathscr{F}_{n}$ 's form a decreasing sequence. Thus, $\bigcap_{\mathscr{F}_{n}}$ is the intersection of a decreasing $\omega$-sequence of prime $z$-filters, and hence is prime $[3,14.2(\mathrm{a})]$. The prime $z$-filter $Z\left[\boldsymbol{O}_{e}\right]$ is containedproperly in $\bigcap_{F_{n}}$. Consider, for example, the full set $A$ such that for each $n \geqq 2, A \bigcap E_{n}^{*}=\varnothing$ for those $E_{n}^{*}$ 's whose points have 0 th coordinate $\leqq n-1$, while $A \bigcap E_{n}^{*}=E_{n}^{*}$ otherwise. Clearly, $A$ belongs to $\bigcap \mathscr{F}_{n}$ but not to $\boldsymbol{Z}\left[\boldsymbol{O}_{e}\right]$. (Incidentally, it is now easy to describe the fallacy in $[4,4.2]$. In effect, it is asserted that because the zero-set $\boldsymbol{Z}(k)$ considered there belongs to $\bigcap \mathscr{F}_{n}$, it belongs to $\boldsymbol{Z}\left[\boldsymbol{O}_{e}\right]$. Actually, there exist functions $k$ satisfying the conditions imposed in $[4,4.2]$ for which $Z(k)$ is the set $A$ just defined.)

Now let $\mathscr{F}$ be a prime $z$-filter contained properly in $\bigcap_{\mathscr{F}_{n}}$, and let $Z \in \bigcap \mathscr{F}_{n}-\mathscr{F}$. We shall show that the $z$-filter generated by $\mathscr{F}$ and $\{Z\}$ is distinct from $\bigcap_{F_{n}}$. By Proposition 2, we may assume that $Z$ is full. Define $T$ to be the set of $\beta$-points in $Z$ of $E_{n}$ 's such that $Z \cap E_{n}^{*}=E_{n}^{*}$. It is easy to see that $T$ is a closed set in $E^{(\omega)}$, whence $T$ is a zero-set. Furthermore, the fact that $Z \in \bigcap \mathscr{F}_{n}$ implies that $T \in \bigcap \mathscr{F}_{n}$ as well. We claim that $T$ does not belong to the $z$-filter generated by $\mathscr{F}$ and $\{Z\}$. Indeed, it suffices to show that if $F \in \mathscr{F}$ has the property that for each $n$, the intersection of $F$ and the union of all the $E_{k}$ 's with $k \leqq n$ is in $\mathscr{B}_{n}$, then $F \cap Z \notin T$. For any $n$, either $F \cap E_{n}^{*} \in \mathscr{U}$ or $F \cap E_{n}^{*}=\varnothing$; and since $Z$ is full, either $Z \cap E_{n}^{*} \notin \mathscr{U}$ or $Z \cap E_{n}^{*}=E_{n}^{*}$. Now $F \in \mathscr{F}$ implies that $F$ meets $E^{(\omega)}-Z$, since $Z \notin \mathscr{F}$. Thus, for at least one integer $n, F$ meets some $E_{n}^{*}$ such that $Z \cap E_{n}^{*} \notin \mathscr{U}$; let $m$ be the smallest such integer. Then for some $E_{m}^{*}$, we have $F \cap E_{m}^{*} \in \mathscr{U}$ and $Z \cap E_{m}^{*} \notin \mathscr{U}$. Suppose that for all such $E_{m}^{*}$, the $\beta$-point of $E_{m}$ did not belong to $Z$. Then, for any $E_{m-1}$ to which such $E_{m}$ 's are attached, we should have $Z \cap E_{m-1}^{*} \notin \mathscr{U}$, since $Z$ is full; but also $F \cap E_{m-1}^{*} \neq \varnothing$, because of the restriction on $F$. This contradicts the choice of $m$. Thus, for some $E_{m}$ such that $Z \cap E_{m}^{*} \notin \mathscr{U}$ and $F \cap E_{m}^{*} \in \mathscr{U}$, the $\beta$-point $p$ belongs to $Z$. Hence $p \in F \cap Z$, but $p \notin T$; so $F \cap Z \notin T$, as required. This shows that $\bigcap \mathscr{F}_{n}$ has no immediate predecessor.

Since $\bigcap \mathscr{F}_{n}$ is a nonminimal prime $z$-filter, the question arises whether the intersection of a decreasing $\omega$-sequence of prime $z$-filters can be minimal, or more strongly, of the form $Z\left[O^{p}\right]$. (By $[2,4.2]$, the latter is equivalent to asking whether there is a space that provides the illustration that was supposed to have been provided by $E^{(\omega)}$, that is, whether there is a $\beta F$-point $p$ such that the positive cone of $C / O^{p}$ has a countable coinitial subset.) The next example shows that the answer to even the stronger question is in the affirmative.

EXAmple 2. A minimal prime $z$-filter of the form $Z\left[O^{p}\right]$ that is the intersection of a decreasing $\omega$-sequence. We define a space $S$ with the same underlying set as $E^{(\omega)}$. A base $\mathscr{B}$ for the neighborhood system of $e$ will consist of the sets of the form $\bigcup B_{n}, B_{n} \in \mathscr{B}_{n}$, where for each $n>0$, the intersection of $B_{n+1}$ and 
the union of all $E_{k}$ 's with $k \leqq n$ is a subset of $B_{n}$; every other point of $S$ will be isolated. Each set in $\mathscr{B}$ is open-and-closed; hence $S$ is a completely regular space. We note that although $S$ has a stronger topology than the extremally disconnected space $E^{(\omega)}$, it is not extremally disconnected; $E_{1}-\{e\}$, for example, is an open set in $S$ whose closure $E_{1}$ is not open in $S$.

Let $\mathscr{G}_{n}$ denote the $z$-filter on $S$ generated by $\mathscr{B}_{n}$. Then the collection of $\mathscr{G}_{n}$ 's is a chain of $z$-filters, and the argument used to show that the $z$-filters $\widetilde{F}_{n}$ of Example 1 are prime shows that the $\mathscr{G}_{n}$ 's are prime. Clearly $Z\left[\boldsymbol{O}_{c}\right] \subset \bigcap_{\mathscr{G}_{n}}$. To see that $Z\left[\boldsymbol{O}_{e}\right]=\bigcap \mathscr{G}_{n}$, observe that, because of the nature of the sets in $\mathscr{B}$, if a zero-set $Z$ in $S$ contains a set in each $\mathscr{B}_{n}$, it contains a set in $\mathscr{B}$, and hence is in $Z\left[\boldsymbol{O}_{e}\right]$. Since the intersection of a chain of prime $z$-filters is prime $[3,14.2(\mathrm{a})], Z\left[\boldsymbol{O}_{e}\right]$ is a minimal prime $z$-filter.

In Example 1, we exhibited a nonminimal prime $z$-filter that is the intersection of a decreasing $\omega$-sequence, and has no immediate predecessor. Example 3 shows that this is not characteristic of nonminimal prime $z$-filters that are the intersection of a decreasing $\omega$-sequence. This indicates that it may be difficult to find general results even about the intersection of an $\omega$-sequence. Before giving the example, we prove a result that provides the key to finding a prime $z$-filter smaller than one that has no immediate successor, in certain very special circumstances.

Proposition 3. Let $Y$ be a metric space, and let $\mathscr{F}$ be a prime z-filter on some zero-set $T \subset Y$. Assume that there exists a disjoint family of relatively openand-closed subsets of $Y-T$ such that for each $p \in T$, there is exactly one set $A_{p}$ in the family that has $p$ as its unique limit point in $T$, and $Y-T=\bigcup_{p \varepsilon T} A_{p}$. Let $\mathscr{U}_{p}$ be a prime $z$-filter on $A_{p}$ converging to $p$, let $\mathscr{V}$ be an ultrafilter on $T$ containing $\mathscr{F}$ such that $\mathscr{F}$ is the family of zero-sets belonging to $\mathscr{V}$, and let $\mathscr{G}$ be the collection of all closed sets containing a set of the form $F \cup \bigcup_{p \varepsilon V} U_{p}$, where $F \in \mathscr{F}, V \in \mathscr{V}, V \subset F$ and $U_{p} \in \mathscr{U}_{p}$. Then $\mathscr{G}$ is a prime $z$-filter on $Y$ contained properly in the $z$-filter on $Y$ generated by $\mathscr{F}$.

Proof. It is easy to see that each set in the collection used to define $\mathscr{G}$ is closed, and hence belongs to $\mathscr{G}$. The verification that $\mathscr{G}$ is a $z$-filter is routine. Now suppose that $Z \cup Z^{\prime} \in \mathscr{G}$, where $Z$ and $Z^{\prime}$ are zero-sets; then $Z \cup Z^{\prime}$ contains a set of the form $F \cup \bigcup_{p \varepsilon V} U_{p}$, where $F \in \mathscr{F}, V \in \mathscr{V}, V \subset F$ and $U_{p} \in \mathscr{U}_{p}$. For each $p \in V$, $\left(Z \cup Z^{\prime}\right) \cap A_{p} \supset U_{p} \in \mathscr{U}_{p}$, whence $Z \cap A_{p} \in \mathscr{U}_{p}$ or $Z^{\prime} \cap A_{p} \in \mathscr{U}_{p}$. Let

and

$$
W=\left\{p \in T: Z \cap A_{p} \in \mathscr{U}_{p}\right\}
$$

$$
W^{\prime}=\left\{p \in T: Z^{\prime} \cap A_{p} \in \mathscr{U}_{p}\right\} .
$$

Then $W \cup W^{\prime}=V$, so either $W \in \mathscr{V}$ or $W^{\prime} \in \mathscr{V}$. Because $Z \cap T \supset W$ and $Z^{\prime} \cap T \supset W^{\prime}$, it follows that either $Z \in \mathscr{G}$ or $Z^{\prime} \in \mathscr{G}$. Since $T \notin \mathscr{G}, \mathscr{G}$ is contained properly in the $z$-filter on $Y$ generated by $\mathscr{F}$. 
EXAmple 3. A prime $z$-filter that is an immediate predecessor of the intersection of a decreasing $\omega$-sequence. We first define a space with the same underlying set as $E^{(\omega)}$, by replacing sets in $\mathscr{U}$ by complements of finite sets in the definition of basic neighborhood. Attach to each point of this space a copy of the one-point compactification of the integers, by identifying the point with the limit point in the one-point compactification. Call the resulting space $W$. Basic neighborhoods are open-and-closed, so $W$ is a completely regular space. Note that as a set, $W$ can be viewed as the set of all $(\omega+1)$-tuples of non-negative integers that have a finite number of nonzero coordinates, consisting at most of a consecutive set beginning with the 0 th, together with the $\omega$ th. For any $x \in W$, we denote the $\alpha$ th coordinate of $x$ by $x_{\alpha}$. Let $T=\left\{x \in W: x_{\omega}=0\right\}$; there is a natural one-to-one correspondence from $E^{(\omega)}$ onto $T$, defined by

$$
\left(p_{1}, p_{2}, \cdots, p_{n}, 0,0, \cdots\right) \rightarrow\left(p_{1}, p_{2}, \cdots, p_{n}, 0,0, \cdots, 0\right) .
$$

For all $n$, let $\mathscr{C}_{n}$ denote the image in $T$ under this correspondence of the collection $\mathscr{B}_{n}$ of subsets of $E^{(\omega)}$ introduced in Example 1 , define $\mathscr{H}_{n}$ to be the $z$-filter on $W$ generated by $\mathscr{C}_{n}$, and set $\mathscr{P}=\bigcap \mathscr{H}_{n}$. The proof that each $\mathscr{H}_{n}$ is prime is analogous to the proof that $\mathscr{F}_{n}$ in Example 1 is prime (where $\mathscr{V}_{n}$ is interpreted as the image of the ultrafilter used before). The $\mathscr{H}_{n}$ 's form a chain of prime $z$-filters, whence $\mathscr{P}$ is a prime $z$-filter on $W$ that is the intersection of a decreasing $\omega$-sequence. We can now obtain a prime $z$-filter on $W$ that is contained properly in $\mathscr{P}$, but is not generated by the image of any prime $z$-filter on $E^{(\omega)}$ containing $Z\left[O_{e}\right]$. In the notation of Proposition 3, for each $p \in T$, we set $A_{p}=\left\{x \in W: x_{n}=p_{n}\right.$ for $n<\omega$ and $\left.x_{\omega}>0\right\}$. Choose $\mathscr{F}$ to be $\bigcap \mathscr{F}_{n}$, where $\mathscr{F}_{n}$ is the trace of $\mathscr{H}_{n}$ on $T$, and each $\mathscr{U}_{p}$ to be the free ultrafilter $\mathscr{U}$ on $A_{p}$; then define $\mathscr{G}$ as in Proposition 3. It follows from the proposition that $\mathscr{G}$ is a prime $z$-filter contained properly in $\mathscr{P}$. Since $T \notin \mathscr{G}$, there cannot be a prime $z$-filter on $E^{(\omega)}$ containing $Z\left[O_{e}\right]$ whose image generates $\mathscr{G}$. Furthermore, by $[2,3.6]$ and Proposition $1, \mathscr{P}$ is the immediate successor of $\mathscr{G}$.

The construction given in Example 3 may also be carried out in the space $\mathbf{R}$ of real numbers (cf. [5], where the error inherited from [4, 4.2] is, of course, to be corrected also). First note that we can realize a copy of the space $W$ in $\mathbf{R}$ by choosing sequences to correspond to the $E_{n}^{*}$ 's that are contained in intervals of length $1 / 3$ of the distance between consecutive points of any sequence corresponding to an $E_{n-1}^{*}$, coming in to the limit point from the left. Since $\Sigma_{n} 1 / 3^{n}=1 / 2$, there is an interval on the right of each point from which a new sequence may be selected. Attaching the new sequences, we have a copy of $W$, and the prime $z$ filters given in Example 3 may be carried over to $\mathbf{R}$ to give bases for prime $z$ filters having the same inclusion properties.

We are now ready to give an example of a decreasing sequence of prime $z$ filters with no countable coinitial subset. The construction is an extension of the one used in Example 3. 
ExAmple 4. A decreasing $\omega_{1}$-sequence of prime $z$-filters. The underlying set of the space $X$ to be considered consists of all $\omega_{1}$-tuples of non-negative integers that have only a finite number of nonzero coordinates, and such that for each ordinal $\alpha$ with no immediate predecessor, the nonzero coordinates indexed by the $\omega$-sequence following $\alpha$ are consecutive and begin with the $\alpha$ th. For each $\alpha<\omega_{1}$, the sequences of all points of $X$ that have the same coordinates before the $\alpha$ th coordinate and zero coordinates after the $\alpha$ th will be denoted generically by $C_{\alpha}$. A basic neighborhood of a point $p \in X$ will now be defined. If $p$ is the point with all coordinates equal to zero, set $\beta=-1$; otherwise let $\beta$ be the index of the last nonzero coordinate of $p$. A basic neighborhood of $p$ will be a certain type of subset of $\left\{x \in X: x_{\gamma}=p_{\gamma}\right.$ for $\left.\gamma \leqq \beta\right\}$. There is a unique $C_{\beta+1}$ such that $p$ is the point with $(\beta+1)$ st coordinate equal to zero. We add all but a finite number of points of this $C_{\beta+1}$. Next, add all but a finite number of points from each of the $C_{\beta+2}$ 's that contain one of the previously selected points of $C_{\beta+1}$. Continue in this way for nonlimit ordinals. For a limit ordinal $\gamma$, add all but a finite number of points of each $C_{\gamma}$ that contains a previously selected point. The set of all points thus obtained forms a basic neighborhood of $p$. Since each basic neighborhood is open-and-closed, $X$ is a completely regular space.

We shall define inductively a decreasing $\omega_{1}$-sequence of prime $z$-filters that converge to the point with all coordinates equal to zero. For $n<\omega$, let $\mathscr{D}_{n}$ be the image under the natural mapping of $E^{(\omega)}$ into $X$ of the families $\mathscr{B}_{n}$ defined in Example 1, and let $\mathscr{P}_{n}$ be the $z$-filter generated by $\mathscr{D}_{n}$. The proof that the $\mathscr{P}_{n}$ 's are a decreasing $\omega$-sequence of prime $z$-filters is analogous to the proof for the $z$-filters $\mathscr{F}_{n}$ in Example 1 (where $\mathscr{V}_{n}$ is interpreted as the image of the ultrafilter used before). Next, if $\alpha<\omega_{1}$ is a limit ordinal, and a decreasing sequence of prime $z$-filters $\mathscr{P}_{\gamma}, \gamma<\alpha$, has been defined, we set $\mathscr{P}_{\alpha}=\bigcap_{\gamma<\alpha} \mathscr{P}_{\gamma}$; then $\mathscr{P}_{\alpha}$ is prime.

Now let $\beta$ be an infinite nonlimit ordinal, and let $W=\left\{x \in X: x_{\gamma}=0\right.$ for $\gamma>\beta\}$. We can write $\beta=\alpha+n$ for some limit ordinal $\alpha$ and some finite ordinal $n$. Let $T$ be the zero-set $\left\{x \in W: x_{\gamma}=0\right.$ for $\left.\gamma \geqq \alpha\right\}$, and $\mathscr{F}$ the trace of $\mathscr{P}_{\alpha}$ on $T$; note that the induction hypothesis implies that $\mathscr{F}$ is a prime $z$-filter on $T$. For each $p \in T$, let $\mathscr{U}_{p}$ be the $z$-filter on $\left\{x \in W: x_{\gamma}=p_{\gamma}\right.$ for $\left.\gamma<\alpha\right\}$ generated by the collection of sets of the form

$$
\left\{x \in W: x_{\gamma}=p_{\gamma} \text { for } \gamma<\alpha \text { and }\left(x_{\alpha}, x_{\alpha+1}, \cdots, x_{\alpha+n-1}, 0,0, \cdots\right) \in D_{n}\right\},
$$

where $D_{n}$ is a set in the collection $\mathscr{D}_{n}$ defined earlier in the example. A proof that $\mathscr{U}_{p}$ is prime may be given using the ideas of the proof in Example 1 that $\mathscr{F}_{n}$ is prime. A base for the $\beta$ th $z$-filter $\mathscr{P}_{\beta}$ will be defined as follows: Let $\mathscr{V}$ be any ultrafilter containing $\mathscr{F}$ such that $\mathscr{F}$ is the family of all zero-sets belonging to $\mathscr{V}$. A typical set in the base consists of all points in some set $F \in \mathscr{F}$, together with a set from each $\mathscr{U}_{p}$ such that $p$ is in a fixed set $V \subset F$ of $\mathscr{V}$. It is easy to see that $\mathscr{P}_{\beta}$ is a $z$-filter.To prove that it is prime, first let $\mathscr{G}$ denote the trace of $\mathscr{P}_{\beta}$ on the metric subspace $W$ of $X$, and observe that $T$ is a zero-set in $W$. Applying Proposition 3 
to the present $\mathscr{F}$ and $\mathscr{G}$, we conclude that $\mathscr{G}$ is prime. Now if $Z \cup Z^{\prime} \in \mathscr{P}_{\beta}$, where $Z$ and $Z^{\prime}$ are zero-sets on $X$, then $\left(Z \cup Z^{\prime}\right) \cap W \in \mathscr{G}$, so either $Z \cap W \in \mathscr{G}$ or $Z^{\prime} \cap W \in \mathscr{G}$. Hence either $Z \in \mathscr{P}_{\beta}$ or $Z^{\prime} \in \mathscr{P}_{\beta}$. Notice that $\mathscr{P}_{\alpha}$ has a base consisting of subsets of $T$, so that $\mathscr{F}$ generates $\mathscr{P}_{\alpha}$ on $X$. Thus, from Proposition 3, we have $\mathscr{P}_{\beta} \subset \mathscr{P}_{\alpha}$. It follows that for each limit ordinal $\alpha$, the family of $z$-filters $\mathscr{P}_{\alpha+n}$, $n<\omega$, is decreasing, provided one uses the same ultrafilter $\mathscr{V}$ for each $n$. Therefore the entire $\omega_{1}$-sequence of $z$-filters can be constructed so that it is decreasing.

Finally, to see that $\bigcap_{\alpha<\omega_{1}} \mathscr{P}_{\alpha}$ has no immediate successor and is not the intersection of a countable number of larger prime $z$-filters, thereby providing an answer to the question of Gillman and Jerison, it suffices to recall that the set of all prime $z$-filters containing $\bigcap_{\alpha<\omega_{1}} \mathscr{P}_{\alpha}$ is a chain $[3,14.8(\mathrm{a})]$.

\section{REFERENCES}

1. L. Gillman and M. Henriksen, Rings of continuous functions in which every finitely generated ideal is principal, Trans. Amer. Math. Soc. 82 (1956), 366-391.

2. L. Gillman and M. Jerison, Quotient fields of residue class rings of function rings, Illinois J. Math. 4 (1960), 425-436.

3. - - Rings of continuous functions, Van Nostrand, Princeton, N. J., 1960.

4. C. W. Kohls, Prime ideals in rings of continuous functions, Illinois J. Math. 2 (1958), 505-536.

5. _- Prime ideals in rings of continuous functions. II, Duke Math. J. 25 (1958), 447-458.

Syracuse UNIVERSITY,

Syracuse, New York 